\title{
Effect of Mindfulness Sessions on Chronic Fatigue Syndrome and Job Satisfaction of Nurses: A Randomized Clinical Trial
}

Nasrin Bonakdari

Islamic Azad University of Malard

Nadereh Mohammadi

Islamic Azad University of Malard

Mohammad Reza Taghvizadeh Yazdi

Islamic Azad University of Malard

Reza Norouzadeh

Shahed University

Mohammad Abbasinia

Qom University of Medical Science and Health Services

Bahman Aghaie ( $\sim$ b.aghaie66@yahoo.com )

Qom University of Medical Science and Health Services

\section{Research Article}

Keywords: Mindfulness, Chronic fatigue syndrome, Job satisfaction, Nurse, Randomized controlled trial

Posted Date: June 7th, 2021

DOI: https://doi.org/10.21203/rs.3.rs-567623/v1

License: (c) (i) This work is licensed under a Creative Commons Attribution 4.0 International License.

Read Full License 


\section{Abstract}

Background: Nursing is considered a stressful profession, so nurses are at higher risk of physical and mental illness. Mindfulness is an important concept for nursing with practical implications for nurse wellbeing, development, and quality nursing care sustainability.

Objectives: This study identified mindfulness sessions' effect on nurses' chronic fatigue syndrome(CFS) and job satisfaction )JS.(

Methods: A randomized clinical trial design was conducted on 80 nurses to intervention and control groups. The intervention in the intervention group was eight mindfulness sessions training, and the control group had no intervention. Nurses' CFS and JS were assessed using the Minnesota Satisfaction Questionnaire and The Multidimensional Fatigue Inventory.

Results: After implementing a mindfulness-based stress reduction program, the intervention group nurses reported lower CFS and higher JS than the control group $(p<0.005)$.

Conclusion: The findings of this study indicate the effectiveness of mindfulness programs on improving nurses' chronic fatigue and JS. Since CFS and job dissatisfaction are two disturbing elements for nurses' optimal performance in professional and personal life, this study can recommend the implementation of mindfulness exercises as a routine, operational and uncomplicated program in nurses.

\section{Introduction}

Mindfulness is an important concept for nursing with practical implications for nurse well-being, development, the sustainability of quality nursing care, and comprehensive health promotion(1). Various studies have been presented on mindfulness's effects that mindfulness reduces stress, distress, negative attitudes and increases adaptive skills $(2,3)$. Song (2015) showed that mindfulness intervention makes students more successful in clinical and academic work due to increased attention and decreased depression, anxiety, and stress(4).

Nursing is considered a stressful profession, so nurses are at higher risk of physical and mental illness. One of the complications of chronic stress is fatigue syndrome or myalgic encephalomyelitis $(5,6)$. CFS is a debilitating disorder that is exacerbated by physical and mental activity and pains that result in decreased mental endurance $(7,8)$. Nurses are often trapped in a cycle of fatigue because of their multiple roles at work and life. This causes them to be exposed to various job-related mistakes, such as medication errors, reduced work efficiency due to physical and mental impairment, memory loss, prolonged reaction time, reduced decision making, and decreased motivation and absenteeism $(9,10)$. In a study of 700 Chinese nurses, Huang (2019) found that married nurses with long work weeks, night shifts, and dissatisfaction with patient communication, excessive commitment, and expectation of reward for greater effort were more affected by chronic fatigue(10). In a study of the dimensions of 
fatigue and burnout, Azmoon (2018) found that nurses reported general, physical, and mental fatigue, which was associated with depersonalization, reduced personal success, and decreased motivation(11).

JS is one of the important variables in promoting nursing care. Nurses' JS is more affected by job stressors and organizational, managerial, professional identity, conflict of professional role, commitment, or interaction with physicians and the environment (12-14). Many factors in the nursing profession, such as time constraints, being in difficult decision situations, and not being prepared to meet patient or family's needs, lead to burnout and physical illness (15). In this regard, nurses' emotional empowerment can help manage JS and stress in the nursing profession. For example, a psychomotor relaxation program has been shown to have a favorable effect on nurses' psychological and physiological status, emotional exhaustion, and depression(16). Other interventions used to reduce nurses' job stress include cognitive-behavioral stress management, which has been confirmed to reduce stress and productivity (17).

Among the psychological approaches, mindfulness is a method based on cognitive behavioral therapy principles that can reduce work stress, improve depression, anxiety, well-being, and work-related burnout in health workers because of increased awareness of stress assessment and a greater understanding of useful behavioral options to respond to stress(18). The well-known mindfulness mechanism is the improvement of emotional adjustment processes by modulating perceived stress and anxiety, enhancing self-awareness, attentiveness, empathy, cognitive flexibility, compassion, and, in general, emotional skills, including emotional acceptance and adjustment of one's well-being(19). Also, it has been shown that mindfulness as an effective factor in psychological well-being plays an important role in self-esteem and life satisfaction(20).

In Iran, the effects of mindfulness intervention have been studied on quality of life and mental health of chemically injured veterans(21), post-traumatic stress disorder in victims of war(22), reduced anxiety, depression, and burden of a caregiver in patients with multiple sclerosis(23), emotional health and glycemic control of diabetic patients(24,25), quality of life in cardiovascular patients(26), drug-dependent male(27), psychological health of elderly women with chronic pain(28), and satisfaction with sexual identity in Iranian adolescents(29). The studies of the effects of mindfulness techniques on nurses include the effectiveness of mindfulness-based cognitive therapy on the cognitive failure and emotional process of anxious nurses(30), emotional maturity, and job stress (30). Also, studies have focused on the predictive role of mindfulness on secondary traumatic stress (31)and nurses' emotional adjustment (32).

Since there was no evidence in Iran that mindfulness could affect nurses' JS and CFS, the authors of this article draw on the clear principle that "take care of yourself so you can take care of others." this message is often overlooked by the professional lives of nurses. Therefore, Nurses' needs are not prioritized, and often lack of self-care increases the harmful effects of stress on nurses, especially in the continually changing clinical environment(33). Due to the importance of nursing physical and mental health in patients' quality care, this study aimed to determine the effect of mindfulness-based stress reduction programs on CFS and JS in nurses. 


\section{Methods}

\subsection{Study design}

This is a single-blind randomized clinical trial in two groups in the Karaj University of Medical Sciences, Alborz, Iran, 2019.

\subsection{The inclusion and exclusion criteria}

Inclusion criteria included at least five years' clinical experience, low JS (score=19-38), and high fatigue (score $\geq 12$ ). Exclusion criteria included not completing the questionnaires and absence during the intervention

\subsection{Participants and Sample size}

The statistical population of this study included all 420 nurses in one hospital. According to Morgan's table, 201 nurses were randomly selected and answered the Chronic Fatigue Syndrome Questionnaire. Eighty participants met the inclusion criteria and were randomly assigned to intervention and control groups.

\subsection{Intervention}

Nurses were randomly assigned to an intervention group. Mindfulness sessions were implemented for eight weeks for nurses in the intervention group. Each session lasted 90 minutes. The training of each session was based on specific objectives and concepts of mindfulness(3). No intervention was performed for the control group. In the intervention group, before the intervention, participants completed Multidimensional Fatigue Inventory (MFI) and the short form of Minnesota Satisfaction Questionnaire (MSQ), and after completing the sessions, the participants completed the questionnaires again. The questionnaires were completed at the same time in the control group. The nurse who participated in the intervention remained blind to participants' assignment in the intervention and control groups until the initial analysis of the results was completed.

\subsection{Measurement}

Data collecting instruments were the MFI and the short form of Minnesota MSQ. MFI was used to measure the level of CFS before and after the intervention. The MFI is a self-administered questionnaire with 20 items with 5 subscales: general fatigue, physical fatigue, mental fatigue, reduced activity, and reduced motivation. Each subscale includes four items with five-point Likert scales. Internal consistency ( $a=0.810-0.952)$, correlations for convergent validity (0.466-0.948), and discriminant validity $(0.121-$ 0.5 ) were reported for all subscales in the Persian Version of MFI (34). Scores on each subscale range from 4 to 20, with higher scores indicating greater fatigue. Higher scores indicate greater severity of fatigue. The short form of the Minnesota Satisfaction Questionnaire (MSQ) is a self-administered questionnaire used to measure JS before and after the intervention. The short form of MSQ has 20 items 
on a 5-point scale (35). A score 19 to 38 show low JS, a score of 38 to 57 indicates average JS and a score above 57 suggest JS is very good. The Persian version of the MSQ shows good validity and reliability for measuring JS (36).

\subsection{Data analysis}

Statistical Package for Social Sciences (SPSS) software Ver. 15.0 was applied to data analysis. The hypothesis of research: mindfulness sessions are effective on CSF, and nurses' JS was analyzed by multivariate analysis of covariance (MANCOVA). The assumptions for this test were examined. Box's M test was used to know the equality of covariance between the groups. Levene's test was used to determine the homogeneity of variances. Also, to determine which of the components of the dependent variables the differences are related to, one-way covariance test was used in MANCOVA text.

\section{Results}

Sixty of the nurses were female, and 20 were male, and the mean age $35 \pm 5$. Clinical experience was $15 \pm 4$ years, and 58 participants were bachelor's degree and 22 with master's degree in nursing. Sixty-six participants were married, and 14 were single. There was no statistically significant difference in demographic characteristics between the intervention and control groups (Table 1).

The first hypothesis of research: mindfulness is effective on nurses' JS was analyzed by MANCOVA. Box's $M$ test did not show the assumption of the covariance matrix's homogeneity; given the equality of the number of groups, we ignored it (Table 2). Levene's test was used to determine the homogeneity of variances. Significant level $(P=0.105)$ indicating that the assumption of homogeneity of variances had been broken in the component of external satisfaction, but this assumption can be ignored considering the equality of the number of groups (Table 3). The results show that by controlling the effect of pre-tests, Wilks Lambda is significant (Table 4). To determine which of the components of the dependent variables the differences are related to, one-way ANCOVA test was used in MANCOVA text. According to the oneway ANCOVA for post-test of JS components, by controlling the effect of pre-tests, the experimental had a significant difference in internal and external satisfaction scores. After the intervention, the mean internal and external satisfaction of the intervention group increased significantly. The results showed in the intervention group 38.4\% (Partial Eta Square= 0.384) of the increase in "internal satisfaction" and $36.5 \%$ (Partial Eta Square=0.365) of the increase in "external satisfaction." This result showed mindfulness sessions had increased JS in nurses (Table 5). Also, findings show that the intervention group's post-test scores on JS were significantly higher than the pre-test score, while the mean change in the control group was not significant (Table 6).

The second hypothesis of research: mindfulness is effective on nurses' CSF was analyzed by MANCOVA. Box's M test did show the assumption of the covariance matrix's homogeneity (Table1). Levene's test was used to determine the homogeneity of variances (Table 7). The F value was significant in all components of CFS $(a=0.01)$. The results show that by controlling the effect of pre-tests, Wilks Lambda is significant (Table 8). To determine which of the components of the dependent variables the differences are related 
to, one-way ANCOVA test was used in MANCOVA text. According to the one-way ANCOVA for post-test of JS components, by controlling the effect of pre-tests, the experimental had a significant difference in components of CFS. The results showed in intervention group 63.4\% (Partial Eta Square $=0.634$ ) of the decrease in "General fatigue ", 69.5\% (Partial Eta Square=0.695) in physical fatigue, 65/4\% (Partial Eta Square $=0.645$ ) in Reduced activity, 67/9\% (Partial Eta Square=0.679) in Reduced activity, 78\% (Partial Eta Square $=0.78$ in reduced motivation. This result showed mindfulness sessions had decreased CFS in nurses (Table 9). The finding showed that CFS in the intervention group decreased after the mindfulness sessions, while the mean change in the control group was not significant (Table 10).

\section{Discussion}

The present study shows the effectiveness of mindfulness sessions on the symptoms of CFS and JS in nurses. Penque (2019) Showed that mindfulness program reduces job burnout and promotes positive stress responses in nurses by strengthening psychological aspects (37). Mindfulness-based programs can help relieve mental and physical fatigue by reducing the heart rate and creating a state of relaxation (38). Reducing mental and physical fatigue following mindfulness programs may increase situational awareness and improve nurses' decision-making(37). The results show after the intervention of mindfulness, internal and external satisfaction show improvement in nurses. This is inconsistent with a descriptive study of Kim (2016) conducted on 71 clinical nurses at Daegu hospital in South Korea; that mindfulness and JS did not have a significant relationship with each other, and self-efficacy was identified as a factor increasing nurses' JS. In any case, the present study did not examine the mediation role of self-efficacy on the effect of mindfulness on nurses' JS. Our study shows the reasons for increasing job satisfaction in nurses are learning and awareness of how to resolve stress and job conflicts after the intervention. In line with this finding, Lin (2020) explores the relationships between nurses' mindfulness and job satisfaction in 1,043 Chinese hospital nurses and shows a medium-high level of mindfulness, whereas job satisfaction among the nurses was at medium levels. Also, job satisfaction was affected by nurses' mindfulness directly(39).

The present study also shows that all CFS components in the intervention group change significantly by controlling the pre-test effect. Mindfulness sessions reduce the incidence of CFS in nurses. This decrease can be seen in general fatigue, physical fatigue, reduced activity, reduced motivation, and mental fatigue, respectively(37). Consistent with the findings of this study, other studies show the effectiveness of mindfulness strategy in nurses. Slatyer et al. (2018) performed mindfulness-based self-care and resiliency (MSCR) to reduce compassion fatigue and enhance flexibility in 20 nurses. The extracted themes show participants' perceptions of the program's benefits for nurses and in routine practice. Gaining perspective and insight; creating a feeling of inner calm; taking time to care for self; feasibility and acceptability of the MSCR program; and using self-care strategies that demonstrate the efficacy of mindfulness program in nurses(40). One of the known benefits of mindfulness in this study is to prevent decreased motivation. In any case, it is not clear what factors reduce motivation and how mindfulness training promotes motivation in nurses. 
Regarding the effect of mindfulness on nursing students, Koren et al. (2017) show that mindfulness training help reduces stress and increase awareness of the moment. Thus, mindfulness by influencing spirituality, as the core of holism, may motivate nurses to find meaning and purpose in life(41). Another finding of this study is that mindfulness intervention reduces mental fatigue in nurses. Similarly, Suleiman - Martos et al. (2020), in a systematic review and meta-analysis of 17 related articles with a sample size of 632 nurses, determine mindfulness has a good effect on nurses' burnout and reduces emotional exhaustion(42). In the present study, all participants with high fatigue scores and aspects of nurses' fatigue are reported in physical and mental areas. As research shows, chronic fatigue as a negative consequence reduces nurses' mental resilience. In this study, the effects of mindfulness training sessions are presented on clinical nurses' physical and mental fatigue. We still do not know if fatigue originates primarily from the nurses' physical aspects or that pre-existing/ concentrated psychoemotional issues in the years of professional work. But what is certain is that mindfulness training has been able to significantly reduce nurses' physical and mental fatigue in a two-month process. In line with the effects of mindfulness on fatigue, Best et al. (2020), in their study of mindfulness-based stress reduction program through a mobile application with a group of military nurses, show reduced burnout in seventy-five percent of nurses participating in this program and a $100 \%$ improvement in their stress and conscious attention. These researchers suggest that mindfulness training is probably one of the best ways for nurses to reduce their symptoms of compassion fatigue(39). Therefore, when we can avoid interventions such as mindfulness, which are often not very time-consuming and are welcomed by many people, we can greatly reduce such complications as a destructive consequence of nurses' burnout.

\section{Conclusions}

This study's findings indicate the effectiveness of mindfulness sessions on improving nurses' CFS and JS. Since CFS and JS are two disturbing elements for nurses' optimal performance in professional and personal life, this study recommends implementing mindfulness training as an operational and uncomplicated program. However, such strategies require multiple sessions of intervention and careful adherence of nurses to the training programs. The authors of this article suggest that future studies analyze the effects of the mindfulness sessions on these variables in many nurses from hospital centers and in different wards.

\section{Abbreviations}

CFS: Chronic Fatigue Syndrome, JS: Job Satisfaction, MFI: Multidimensional Fatigue Inventory MSQ: Minnesota Satisfaction Questionnaire, SPSS: Statistical Package for Social Sciences MANCOVA: Multivariate Analysis of Covariance

\section{Declarations}

\section{Ethics approval and consent to participate}


Ethical approval was from the Ethics Committee of Qom University of Medical Sciences (No.

IR.MUQ.REC.1399.307). All nurses provided the written informed consent, and the study's purpose was informed to them. The researcher assured nurses of the voluntary participation, anonymity, and right to withdraw from the study at any time. We confirm that all methods were performed in accordance with the relevant guidelines and regulations.

\section{Consent for publication}

Not applicable

\section{Availability of data and materials}

All data generated and analyzed included in this article.

\section{Competing interests}

The authors have no competing interests to declare.

\section{Funding}

The authors have not received any funding.

\section{Authors' contributions}

NB and BA: Conceptualization, Methodology, Writing- Original draft preparation, and Editing

RN, BA and NM: Conceptualization, Methodology, Data curation, Validation, and Editing

MRTY, NB and MA: Methodology, Supervision, Data curation, Visualization, Investigation, Validation

\section{Acknowledgements}

The authors would like to thank the Research Administration of Qom University of Medical Sciences and all nurses who participated in the study.

\section{References}

1. White L. Mindfulness in nursing: An evolutionary concept analysis. Journal of advanced nursing. 2014;70(2):282-94.

2. Beddoe AE, Murphy SOJJoNE. Does mindfulness decrease stress and foster empathy among nursing students? 2004;43(7):305-12.

3. Song $Y$, Lindquist RJNet. Effects of mindfulness-based stress reduction on depression, anxiety, stress and mindfulness in Korean nursing students. 2015;35(1):86-90. 
4. Song Y, Lindquist R. Effects of mindfulness-based stress reduction on depression, anxiety, stress and mindfulness in Korean nursing students. Nurse education today. 2015;35(1):86-90.

5. Kara IH, Demir D, Erdem Ö, Sayin GT, Yildiz N, Yaman H. Chronic Fatigue Syndrome among nurses and healthcare workers in a research hospital in Turkey. Social Behavior and Personality: an international journal. 2008;36(5):585-90.

6. Bush M. Chronic fatigue syndrome: What nurses need to know. Nursing2020. 2020;50(4):50-4.

7. Roberts D. Diagnosing and managing chronic fatigue syndrome. Nursing in Practice. 2016;89.

8. Friedman KJ, Mattey B, Newton F. School Nurses Can Improve the Lives of Students With Myalgic Encephalomyelitis/Chronic Fatigue Syndrome. NASN School Nurse. 2018;33(6):372-9.

9. Sharma A, Verma A, Malhotra D. Job Performance and chronic fatigue syndrome in nurses. Asian Social Science. 2010;6(12):167.

10. Huang H, Liu L, Yang S, Cui X, Zhang J, Wu H. Effects of job conditions, occupational stress, and emotional intelligence on chronic fatigue among Chinese nurses: a cross-sectional study.

Psychology research and behavior management. 2019;12:351.

11. Azmoon H, Nodooshan HS, Jalilian H, Choobineh A, Shouroki FK. The relationship between fatigue and job burnout dimensions in hospital nurses. Health Scope. 2018;7(2).

12. Mousazadeh S, Yektatalab S, Momennasab M, Parvizy S. Job Satisfaction Challenges Of Nurses In The Intensive Care Unit: A Qualitative Study. Risk Management and Healthcare Policy. 2019;12:233.

13. Zangaro GA, Soeken KL. A meta-analysis of studies of nurses' job satisfaction. Research in nursing \& health. 2007;30(4):445-58.

14. Lu H, Barriball KL, Zhang $X$, While AE. Job satisfaction among hospital nurses revisited: a systematic review. International journal of nursing studies. 2012;49(8):1017-38.

15. Mathew NA. Effect of Stress on job satisfaction among nurses in central kerala. Journal of Business and Management. 2013;7(2):47-51.

16. Veiga G, Rodrigues AD, Lamy E, Guiose M, Pereira C, Marmeleira J. The effects of a relaxation intervention on nurses' psychological and physiological stress indicators: A pilot study. Complementary therapies in clinical practice. 2019;35:265-71.

17. Shariatkhah J, Farajzadeh Z, Khazaee K. The Effects of Cognitive-Behavioral Stress Management on Nurses' Job Stress. Iranian journal of nursing and midwifery research. 2017;22(5):398.

18. Strauss C, Gu J, Pitman N, Chapman C, Kuyken W, Whittington A. Evaluation of mindfulness-based cognitive therapy for life and a cognitive behavioural therapy stress-management workshop to improve healthcare staff stress: study protocol for two randomised controlled trials. Trials. 2018;19(1):209.

19. Barattucci M, Padovan AM, Vitale E, Rapisarda V, Ramaci T, De Giorgio A. Mindfulness-Based IARA Model $B$ Proves Effective to Reduce Stress and Anxiety in Health Care Professionals. A Six-Month Follow-Up Study. International journal of environmental research and public health. 2019;16(22):4421. 
20. Pepping CA, O’Donovan A, Davis PJ. The positive effects of mindfulness on self-esteem. The Journal of Positive Psychology. 2013;8(5):376-86.

21. Arefnasab Z, Babamahmoodi A, Babamahmoodi F, Noorbala AA, Alipour A, Panahi Y, et al. Mindfulness-based stress reduction (MBSR) and its effects on psychoimmunological factors of chemically pulmonary injured veterans. Iranian Journal of Allergy, Asthma and Immunology. 2016;15(6):476-86.

22. Marzabadi EA, Zadeh SMH. The Effectiveness of Mindfulness Training in Improving the Quality of Life of the War Victims with Post Traumatic stress disorder (PTSD). Iranian journal of psychiatry. 2014:228-36.

23. Khazaeili M, Zargham Hajebi M, Mohamadkhani P, Mirzahoseini H. The Effectiveness of Mindfulness-Based Intervention on Anxiety, Depression and Burden of Caregivers of Multiple Sclerosis Patients Through Web Conferencing. Practice in Clinical Psychology. 2019;7(1):21-32.

24. Armani Kian A, Vahdani B, Noorbala AA, Nejatisafa A, Arbabi M, Zenoozian S, et al. The Impact of Mindfulness-Based Stress Reduction on Emotional Wellbeing and Glycemic Control of Patients with Type 2 Diabetes Mellitus. Journal of diabetes research. 2018;2018:1986820.

25. Rahmani S, Zahirrodin A, Moradi M, Hoveida S, Nejati SJIJoD, Obesity. Examining the effectiveness of mindfulness-based stress reduction program and conscious yoga on quality of life in patients with diabetes type 2. 2014;6(4):168-75.

26. Momeni J, Omidi A, Raygan F, Akbari H. Comparing the Effects of Mindfulness-Based Stress Reduction and Cognitive Behavioral Therapy on the Quality of Life of Patients with Cardiovascular Disease. International Journal of Body, Mind and Culture. 2018;5(2):94-103.

27. AsI NRH, Hosseinalipour F. Effectiveness of mindfulness-based stress reduction intervention for health-related quality of life in drug-dependent males. Iranian Red Crescent medical journal. $2014 ; 16(9)$.

28. Alemi S, Malihialzackerini S, Abolmaali Alhoseini K, Khabiri M. Comparison of the Effectiveness of Mindfulness Training and Massage-Aromatherapy on Rising Psychological Health of Elderly Women with Chronic Pain. Iranian Journal of Health Education and Health Promotion. 2019;7(4):359-70.

29. Azarkhordad F, Jenaabadi H, Mehdinezhad V. The Effectiveness of Sex Education Based on Mindfulness Training and Islamic Teachings in Improving Satisfaction with Sexual Identity Among Male Adolescents in Zahedan, Iran. Jundishapur Journal of Health Sciences. (In Press).

30. Jalili Nikoo S, Ghasemi Jobaneh R, Taghvaeinia A. Effectiveness of Mindfulness Training on Emotional Maturity and Occupational Stress of Nurses. Report of Health Care. 2018;4(1):20-5.

31. Ariapooran S. The Prevalence of Secondary Traumatic Stress among Nurses in Iran, Malayer: The Predicting Role of Mindfulness and Social Support \%J International Journal of Community Based Nursing \& Midwifery. 2013;1(3):156-64.

32. Babaei Nadinluye K, Mikaeli Manee F, Pezeshki H, Bafande H, Abdi H. Relationship between mindfulness and meta-emotion on predicting emotional adjustment of novice nurses. Iran Journal of Nursing. 2017;30(105):11-22. 
33. Halm M. The role of mindfulness in enhancing self-care for nurses. American Journal of Critical Care. 2017;26(4):344-8.

34. Daneshmandi H, Choobineh A, Ghaem H. Psychometric properties of the persian version of the "Multidimensional Assessment of Fatigue Scale". International journal of preventive medicine. 2019;10.

35. Spector PE. Job satisfaction: Application, assessment, causes, and consequences: Sage publications; 1997.

36. Mousazadeh S, Yektatalab S, Momennasab M, Parvizy S. Job satisfaction and related factors among Iranian intensive care unit nurses. BMC research notes. 2018;11(1):823.

37. Penque S. Mindfulness to promote nurses' well-being. Nursing management. 2019;50(5):38.

38. Amutio A, Martínez-Taboada C, Hermosilla D, Delgado LC. Enhancing relaxation states and positive emotions in physicians through a mindfulness training program: a one-year study. Psychology, health \& medicine. 2015;20(6):720-31.

39. Best NI, Durham CF, Woods-Giscombe C, Waldrop J. Combating Compassion Fatigue With Mindfulness Practice in Military Nurse Practitioners. The Journal for Nurse Practitioners. 2020;16(5):e57-e60.

40. Slatyer S, Craigie M, Rees C, Davis S, Dolan T, Hegney D. Nurse experience of participation in a mindfulness-based self-care and resiliency intervention. Mindfulness. 2018;9(2):610-7.

41. Koren ME. Mindfulness interventions for nursing students: Application of modelling and role modelling theory. International Journal of Caring Sciences. 2017;10(3):1710-6.

42. Suleiman-Martos N, Gomez-Urquiza JL, Aguayo-Estremera R, Cañadas-De La Fuente GA, De La Fuente-Solana El, Albendín-García L. The effect of mindfulness training on burnout syndrome in nursing: A systematic review and meta-analysis. Journal of advanced nursing. 2020;76(5):1124-40.

\section{Tables}

Table1. Characteristics of Participants $(\mathrm{N}=\mathbf{8 0})$ 


\begin{tabular}{|lll|}
\hline Frequency/mean \pm SD & Characteristics \\
\hline $35 \pm 5$ & & Age \\
\hline $15 \pm 4$ & Male & Clinical experience \\
\hline 20 & Female & \\
\hline 60 & Bachelor's degree & Education level \\
\hline 58 & Master's degree & \\
\hline 22 & Single & Work setting \\
\hline 14 & Married & \\
\hline 66 & & \\
\hline
\end{tabular}

Table 2. Box's M test for the homogeneity of variance of groups in the JS

\begin{tabular}{|llllll|}
\hline JS & $\mathbf{F}$ & $\mathbf{d f 1}$ & $\mathbf{d f 2}$ & Box's M & P-value \\
\cline { 2 - 6 } & $2 / 63$ & 1 & 259920 & $8 / 36$ & $0 / 048$ \\
\hline CFS & $0 / 887$ & 1 & 5814 & $15 / 5$ & $0 / 057$ \\
\hline
\end{tabular}

Table 3. Levene's test for the homogeneity of variance of groups in the JS

\begin{tabular}{|lllll|}
\hline JS & F & df1 & df2 & P-value \\
\hline Internal satisfaction & $2 / 76$ & 1 & 38 & $0 / 105$ \\
\hline Eternal satisfaction & $8 / 74$ & 1 & 38 & $0 / 005$ \\
\hline
\end{tabular}

Table 4. MANCOVA test for the JS

\begin{tabular}{|lllllll|}
\hline JS & $\mathbf{F}$ & $\mathbf{d f 1}$ & $\mathbf{d f 2}$ & Wilks Lambda & $\mathbf{h}^{2}$ & P-value \\
\hline $28 / 11$ & 2 & 35 & $0 / 384$ & $0 / 661$ & $0 / 001$ \\
\hline
\end{tabular}

Table 5. One-way ANCOVA test for post-test JS components 


\begin{tabular}{|lllllll|}
\hline Group & Variable & $\begin{array}{l}\text { Sum of } \\
\text { squares }\end{array}$ & $\begin{array}{l}\text { Mean } \\
\text { Square }\end{array}$ & F & $\begin{array}{l}\text { P- } \\
\text { value }\end{array}$ & $\begin{array}{l}\text { Partial eta } \\
\text { squared }\end{array}$ \\
& $\begin{array}{l}\text { internal } \\
\text { satisfaction }\end{array}$ & 40.7 & 40.7 & 22.4 & $<$ & 0.384 \\
& $\begin{array}{l}\text { external } \\
\text { satisfaction }\end{array}$ & 21.8 & 21.8 & 20.6 & 0.001 & 0.365 \\
Error & $\begin{array}{l}\text { internal } \\
\text { satisfaction }\end{array}$ & 65.3 & 1.81 & & & \\
& $\begin{array}{l}\text { external } \\
\text { satisfaction }\end{array}$ & 37.9 & 1.05 & & & \\
\hline
\end{tabular}

Table 6. Comparison of JS before and after intervention

\begin{tabular}{|llll|}
\hline JS & Time & $\begin{array}{l}\text { Intervention group } \\
\text { (Mean } \pm \text { SD) }\end{array}$ & $\begin{array}{l}\text { Control group } \\
\text { (Mean } \pm \text { SD) }\end{array}$ \\
\hline \multirow{2}{*}{ Internal satisfaction } & Before & $53.1 \pm 5.65$ & $52.05 \pm 6.49$ \\
\cline { 2 - 4 } & After & $55 \pm 5.71$ & $51.9 \pm 6.31$ \\
\hline \multirow{2}{*}{ Eternal satisfaction } & Before & $25.9 \pm 3.08$ & $24.8 \pm 4.76$ \\
\cline { 2 - 4 } & After & $27.4 \pm 2.66$ & $24.9 \pm 4.67$ \\
& Before & $39.5 \pm 4.36$ & $38.4 \pm 5.62$ \\
\cline { 2 - 4 } & After & $41.2 \pm 4.18$ & $38.5 \pm 5.49$ \\
\hline
\end{tabular}

Table 7. Levene's test for the homogeneity of variance of groups in the CFS

\begin{tabular}{|lllll|}
\hline CFS & F & df1 & df2 & P-value \\
\hline General fatigue & $2 / 1$ & 1 & 38 & $0 / 155$ \\
\hline Physical fatigue & $1 / 21$ & 1 & 38 & $0 / 278$ \\
\hline Reduced activity & $0 / 198$ & 1 & 38 & $0 / 659$ \\
\hline Reduced motivation & $2 / 64$ & 1 & 35 & $0 / 112$ \\
\hline Mental fatigue & $1 / 94$ & 1 & 38 & $0 / 172$ \\
\hline
\end{tabular}


Table8. MANCOVA test for the CFS

\begin{tabular}{|lllllll|}
\hline CFS & F & df1 & df2 & Wilks Lambda & h $^{2}$ & P-value \\
\cline { 2 - 6 } & $66 / 7$ & 5 & 29 & $0 / 008$ & $0 / 92$ & $0 / 001$ \\
\hline
\end{tabular}

Table 9. One-way covariance test for post-test CFS components

\begin{tabular}{|c|c|c|c|c|c|c|c|}
\hline & Variable & $\begin{array}{l}\text { Sum of } \\
\text { squares }\end{array}$ & Df & $\begin{array}{l}\text { Mean } \\
\text { Square }\end{array}$ & $F$ & $\begin{array}{l}\mathrm{P}- \\
\text { value }\end{array}$ & $\begin{array}{l}\text { Partial eta } \\
\text { squared }\end{array}$ \\
\hline \multirow[t]{5}{*}{ Group } & General fatigue & $37 / 8$ & 1 & $37 / 8$ & $57 / 2$ & 0.001 & $0 / 634$ \\
\hline & Physical fatigue & $44 / 9$ & 1 & $44 / 9$ & $75 / 1$ & 0.001 & $0 / 695$ \\
\hline & Reduced activity & $57 / 5$ & 1 & $57 / 5$ & $62 / 2$ & 0.001 & $0 / 654$ \\
\hline & $\begin{array}{l}\text { Reduced } \\
\text { motivation }\end{array}$ & $76 / 4$ & 1 & $76 / 4$ & $69 / 7$ & 0.001 & $0 / 679$ \\
\hline & Mental fatigue & $76 / 06$ & 1 & $76 / 06$ & $116 / 6$ & 0.001 & $0 / 78$ \\
\hline \multirow[t]{5}{*}{ Error } & General fatigue & $21 / 8$ & 33 & $0 / 661$ & & & \\
\hline & Physical fatigue & $19 / 7$ & 33 & $0 / 598$ & & & \\
\hline & Reduced activity & $30 / 5$ & 33 & $0 / 924$ & & & \\
\hline & $\begin{array}{l}\text { Reduced } \\
\text { motivation }\end{array}$ & $36 / 1$ & 33 & $1 / 09$ & & & \\
\hline & Mental fatigue & $21 / 5$ & 33 & $0 / 652$ & & & \\
\hline
\end{tabular}

Table10. CFS in before and after intervention 


\begin{tabular}{|llll|}
\hline CFS & Time & \multicolumn{1}{l}{$\begin{array}{l}\text { Intervention group } \\
\text { (Mean } \pm \text { SD) }\end{array}$} & $\begin{array}{l}\text { Control group } \\
\text { (Mean } \pm \text { SD) }\end{array}$ \\
\hline General Fatigue & Before & $18.2 \pm 5.65$ & $17.6 \pm 2.41$ \\
\cline { 2 - 4 } & After & $16.6 \pm 1.69$ & $18.1 \pm 2.13$ \\
\hline Physical Fatigue & Before & $17.6 \pm 1.95$ & $17.1 \pm 2.44$ \\
\cline { 2 - 4 } & After & $15.8 \pm 1.84$ & $17.5 \pm 2.35$ \\
\hline Ratigue Mental & Before & $17.1 \pm 2.56$ & $16.7 \pm 3.13$ \\
\cline { 2 - 4 } & After & $15.3 \pm 2.07$ & $17.8 \pm 2.77$ \\
\hline Reduced & Before & $16.8 \pm 2.05$ & $16.2 \pm 2.17$ \\
\cline { 2 - 4 } Motivation & After & $15.05 \pm 2.06$ & $17.4 \pm 2.55$ \\
\hline Total & After & $17.1 \pm \pm 2.12$ & $17.6 \pm 2.64$ \\
\cline { 2 - 4 } & Before & $17.52 \pm 2.1$ & $17.05 \pm 1.95$ \\
\cline { 2 - 4 } & After & $15.77 \pm 1.95$ & $17.81 \pm 2.28$ \\
\hline
\end{tabular}

